\title{
Ulrich Beck (1944-2015) et la société mondiale du risque
}

Beck and the world risk society

\section{Stéphane Callens}

\section{(2) OpenEdition}

1 Journals

\section{Édition électronique}

URL : http://journals.openedition.org/developpementdurable/10785

DOI : 10.4000/developpementdurable.10785

ISSN : 1772-9971

Éditeur

Association DD\&T

\section{Référence électronique}

Stéphane Callens, «Ulrich Beck (1944-2015) et la société mondiale du risque », Développement durable et territoires [En ligne], Vol.6, $n^{\circ} 1$ | Mars 2015, mis en ligne le 31 mars 2015, consulté le 10 décembre 2020. URL : http://journals.openedition.org/developpementdurable/10785 ; DOI : https://doi.org/ 10.4000/developpementdurable.10785

Ce document a été généré automatiquement le 10 décembre 2020.

Développement Durable et Territoires est mis à disposition selon les termes de la licence Creative Commons Attribution - Pas d'Utilisation Commerciale 4.0 International. 


\title{
Ulrich Beck (1944-2015) et la société mondiale du risque
}

Beck and the world risk society

\author{
Stéphane Callens
}

1 Ulrich Beck est décédé le $1^{\text {er }}$ janvier 2015 d'un infarctus, à l'âge de 70 ans. Il était un des sociologues les plus marquants de notre époque, directeur jusqu'en 2009 de l'Institut de Sociologie de l'Université de Munich. Il avait fait sa thèse en 1977 sur la profession (Beruf), se situant dans la postérité institutionnelle de Max Weber. Ses thèmes de prédilection étaient le risque, la globalisation, et le lien social contemporain - à superposer et à comparer aux vues de son prédécesseur Max Weber.

2 Ulrich Beck est né en 1944 à Słupsk en Poméranie, aujourd'hui en Pologne. Il était marié à la sociologue Elisabeth Gernsheim, et enseignait à Munich, Paris (MSH) et Londres (London School of Economics and Political Science). Il était conseiller de la région de Bavière, membre de la commission éthique chargée de surveiller la sortie du nucléaire en Allemagne et du Groupe Spinelli (Guy Verhofstadt, Daniel Cohn-Bendit, Jacques Delors, Joschka Fischer, Amartya Sen, Pat Cox) créé pour rappeler aux responsables des pays européens qu'ils sont en charge de l'ensemble de l'Union Européenne. À ce titre, Ulrich Beck avait récemment reproché à Angela Merkel le contraste entre la bienveillance social-démocrate de sa politique interne et la brutalité de sa politique hors des circonscriptions électorales allemandes. Beck a incarné l'option européenne devant la politique unilatérale des États-Unis après les attentats du 11 septembre 2001, ou des choix politiques déterminés seulement par des considérations domestiques, comme il le reprochait à Angela Merkel. Il était favorable à une extension du système Erasmus à l'ensemble des jeunes européens, ainsi qu'à une inclusion sociale qui ne se limite pas au travail salarié, mais part de la seule citoyenneté. 


\section{Le Risque}

3 La thèse de La Société du risque a été exposée par Ulrich Beck dans son ouvrage de 1986, publié au moment de la catastrophe de Tchernobyl. Le risque, chez Beck, est une anticipation d'une catastrophe. Le risque est devenu la mesure de notre action, il est une transformation de la modernité. Tout comme chez Comte, Beck propose une sécularisation des sociétés en deux étapes. Chez Comte, il y avait une étape intermédiaire « métaphysique » avant l'âge positiviste. Pour Beck, cette étape intermédiaire est celle de la transition par une société industrielle. Cette dernière n'est qu'une semi-démocratie avec des idéaux de progrès qui contreviennent aux règles fondamentales de cette même démocratie. Dans la "Société mondiale du risque", les directions que prennent les évolutions et les résultats de la mutation technique deviennent objets de débats, et sont sommées d'être légitimées ( La Société du risque, p. 405 de l'édition française de 2008). Des chocs technologiques plus fréquents font que le potentiel de modelage de la société a quitté le système politique pour s'installer du côté du système sous-politique de la modernisation scientifico-technico-économique. Un exemple de cette "sous-politique » peut être donné par la lutte contre la pollution atmosphérique dans les grandes métropoles : l'extension du réseau routier dépend d'un service technique, alors même que le consensus politique autour de la lutte contre la pollution atmosphérique est réel. Un urbanisme comme celui de Los Angeles avec ses multiples autoroutes résulte uniquement du système sous-politique de la modernisation scientifico-technico-économique. Chez Fukuyama, il faudrait un retour du politique pour faire cesser ce désordre issu des sciences. Mais, les élus, même à Los Angeles, ne sont pas restés inactifs, simplement ils n'ont pu agir que de façon limitée, par exemple par la réglementation favorisant les véhicules Zéro Émission. Beck avance quant à lui un processus de transformation du politique largement indéterminé, mais passant aussi par le canal d'une politique extra-parlementaire. Des décisions à portée politique sont prises par de multiples canaux, par exemple par les entreprises ou des groupes nonparlementaires qui contournent toutes les procédures sous un contrôle parlementaire. Les forums centraux de la sous-politique sont les médias et les tribunaux, non les assemblées parlementaires. La sous-politique se réfère directement aux droits fondamentaux, et joue ainsi un rôle de limitation des pouvoirs politiques institués par voie électorale. Par exemple, dit Beck, la médecine est une sous-politique qui regroupe recherche, formation et pratique, indépendamment de tout assentiment extérieur. Il n'existe pas de séparation de pouvoirs, ni plus généralement la recherche d'un équilibre des pouvoirs. Une caractéristique de la sous-politique est donc son constitutionnalisme incomplet. En particulier, La reconnaissance des droits fondamentaux ne s'accompagne pas nécessairement d'un bon équilibrage des pouvoirs dans les sphères familiale, organisationnelle et scientifique. Dans la Société du risque, Beck insiste sur la restriction de la place des procédures de la démocratie parlementaire par des réseaux sous-jacents qui sont les vrais opérateurs politiques, en ce sens qu'ils mettent en œuvre les transformations importantes qui affectent la vie de tout un chacun. La terminologie de sous-politique peut recouvrir aussi des situations dangereuses, comme des circuits de décisions technocratiques, ou de décisions d'organisations criminelles. Dans la Société $d u$ risque, la montée de la sous-politique peut générer de nouveaux risques politiques. Dans les courants institutionnalistes, ce sont des risques politiques induits par l'activité parlementaire qui sont pris en compte de façon privilégiée. Des dispositions de source parlementaire favorisant les subprimes fournissent un exemple de ces risques politiques 
parlementaires. Pour Hayek, il importe de favoriser le Droit des juges pour éviter ce type de risque d'origine parlementaire. Pour Beck, les risques politiques peuvent être aussi d'origine extra-parlementaire, ce qui est indéniable.

Le propos premier de Beck est que la modernité à une capacité de se transformer. Contrairement à Weber et Fukuyama, la société industrielle ne constitue pas un état final de la modernisation. Une sécularisation des sociétés se poursuit, un processus de civilisation est en cours à travers la globalisation. La sociologie du début du $20^{\mathrm{e}}$ siècle insistait sur l'inexorable rationalisation de la société : ce n'est absolument pas la thèse de Beck qui insiste sur le caractère transitoire de la société de plus en plus taylorisée et contrôlée décrite par Weber, celle de la première modernisation et de l'industrialisation comme la connaissait l'Allemagne de l'époque. Aujourd'hui, la Société mondiale du risque émerge avec la mise sur la place publique de maux endogènes qui ont résisté à tout contrôle.

Beck propose un jeu d'équilibrage des pouvoirs et des contre-pouvoirs. Il s'agit d'établir des garanties juridiques de certaines possibilités d'influence de la sous-politique. Tribunaux, contre-expertises, médias : les combinaisons des pouvoirs doivent permettre d'élaborer une démocratie réflexive particulièrement pour les risques technologiques et naturels. Un contre-pouvoir est nécessaire pour l'expertise technique. Pour la recherche, une gestion des risques en amont doit être impulsée, et le cloisonnement disciplinaire combattu.

6 L'institutionnalisme soulignant les risques issus d'une procédure parlementaire, il serait paradoxal dans ce cadre d'analyse d'amplifier le rôle des parlements. Mais jouer l'expansion de la sous-politique présente sans doute encore de plus grands risques que ceux provenant d'une indiscipline parlementaire. Beck propose plus une démarche que des solutions institutionnelles normatives. L'humanité forme une communauté cosmopolite qui partage les mêmes risques globaux, cependant, les trajectoires de cosmopolitisation sont différentes et doivent prendre en compte les attachements nationaux, médiateurs entre l'individu et le global.

7 La question du changement climatique illustre bien les difficultés à définir cette nouvelle politique du risque que Beck évoquait dès 1986. Cependant, les prédictions théoriques de Beck se sont révélées pertinentes : il y a bien eu une extension de l'insécurité chez tous les individus, ce qui est une des caractéristiques d'une société mondiale du risque. Les réponses apportées en une formulation de première modernisation, comme a pu l'être celle des États-Unis après le 11 septembre, se sont révélées inadaptées. Il reste à mieux définir les formes de société politique ayant des composantes parlementaires et extraparlementaires qui conviennent à une société mondiale du risque, ce à quoi Beck s'employait encore récemment pour la question du changement climatique, par exemple.

\section{La Globalisation}

Beck indique que le monde se transforme par l'apparition d'une conscience de l'appartenance multiple (Qu'est-ce que le cosmopolitisme?, p. 142). Le « cosmopolitisme » est le vocable savant donné à une réflexion politique englobant l'ensemble de l'humanité. La pensée allemande a introduit avec Kant le cosmopolitisme avec un raisonnement téléologique : puisque l'état final de l'humanité est la paix, tous les pédagogues doivent dès maintenant enseigner cette paix. Au XIX ${ }^{e}$ siècle, le cosmopolitisme pouvait ainsi 
apparaître comme un horizon idéal, tandis qu'aujourd'hui, ce qui est réel c'est une altérité qui est toujours proche. La vie quotidienne est maintenant celle d'un cosmopolitisme évident, si bien qu'elle entre de plus en plus en contradiction avec les catégories de compréhension de la réalité qui obéissent à des normes nationales (p. 146). Même les formes de résistance à la mondialisation s'expriment dans une mondialisation politique, et participent donc de la même dynamique.

Beck définit la période présente comme un nouveau processus de civilisation qui opère au niveau de l'individu, et de chaque État. Norbert Elias avait introduit cette notion de processus de civilisation qui réalise une baisse progressive de la violence sociale et l'avait étudié dans une comparaison des petits États européens de la Renaissance. Beck distingue la globalisation comme processus de civilisation du discours politique du multiculturalisme. Beck souligne dans la théorie du multiculturalisme des points de faiblesse. Cette théorie ne s'aperçoit pas de la dynamique de civilisation, et se limite à des groupes à l'intérieur d'un seul pays. Beck voit la globalisation d'une façon plus large, comme la fin des sociétés fermées et des affiliations exclusives pour les individus. Le cosmopolitisme a vocation à être intériorisé au plan étatique (p. 344). L'ensemble des pays doivent coopérer pour prendre soin des individus, tous aux multiples affiliations. Beck a proposé sans doute la théorie la plus aboutie à propos de la globalisation vécue à l'époque contemporaine.

\section{Le Lien social}

Beck développe une vision de la catastrophe émancipatrice, produisant de la norme sociale et du bien public. Dans les débuts de la sociologie au XIX ${ }^{e}$ siècle, Comte suppose que toute agglomération humaine apporte la sociabilité. Du côté des socialismes politiques qui apparaissent à la même époque, une Révolution sociale doit venir apporter dans un temps proche les régulations d'une époque de paix et de justice. Aujourd'hui, une crise environnementale va naître par exemple d'un épisode de smog urbain: comme chez Comte, cela veut dire que l'agglomération apporte quelque chose de positif, le fait que la concentration urbaine permet de créer une prise de conscience environnementale. Les socialismes révolutionnaires placent quant à eux la catastrophe positive à l'intérieur d'un avènement volontaire. Mais la création de nouvelles régulations provient en fait de la catastrophe émancipatrice initiale, selon Beck. Par exemple, le risque climatique a généré des communautés cosmopolites d'acteurs (Beck et al., 2013). Le propos de Beck est méthodologique : pour former des politiques appropriées aux problèmes globaux tels que le changement climatique, il oppose un cosmopolitisme méthodologique au nationalisme méthodologique des sociologues. Le nationalisme des communautés est instauré à l'époque des migrations européennes vers le Nouveau Monde par un imaginaire créole, où des résidents coloniaux se trouvent entre une métropole qui décide, et des nouveaux arrivants ou nouveaux compétiteurs. Le nationalisme méthodologique de la plupart des sociologues ne peut qu'introduire des vues trop partielles sur une question comme le changement climatique. Les méthodes de la sociologie doivent donc être adaptées à la situation présente, ce qui revient à adopter un cosmopolitisme méthodologique.

11 Dans la sociologie de Beck, la globalisation (ou réalisme cosmopolite) est un fait : chacun à plusieurs affiliations, et il n'y a plus d'Orient lointain, mais chacun a un restaurant japonais ou libanais comme voisin. De même, le risque climatique donne un retentissement global à de petits gestes quotidiens. 
12 Selon Beck, les cultures de la soutenabilité oscillent entre l'utopie (une tradition dans la lignée de la philosophie idéaliste d'Hegel) et l'apocalypse (généralement invoqué pour réclamer un état d'urgence, une réglementation avec une main de fer). En pratique, les apocalyptiques (par exemple, des groupes terroristes) jouent l'intensification des conflits locaux et poussent à une politique de tension et de législation d'exception. Les territoires ayant aujourd'hui la capacité de reproduire les conflits globaux à petite échelle, sont entrânés par ces politiques à grande échelle.

13 La culture positive du changement climatique, celle de la catastrophe émancipatrice selon Beck, présente les caractéristiques suivantes :

- Elle revendique une connaissance limitée ;

- Elle se place dans une temporalité intermédiaire entre des cultures utopiques, généralement techniciennes, qui sont atemporelles ou dans des horizons de temps très long; et des cultures de l'urgence politique, avec des horizons si courts qu'il n'y a plus d'après-demain. Cette temporalité intermédiaire est celle de l'intergénérationnel court (celui de nos enfants et de nos petits enfants).

14 Il distingue trois types d'acteurs dans la culture positive du changement climatique :

- Les clubs de villes durables, la planification territoriale; par exemple, l'ICLEI, une association de 1200 grandes villes dans le monde. L'ICLEI est assez élitiste - New York, Paris - etc. Les grandes métropoles en font partie ;

- Les consortiums des réseaux d'innovation, les entrepreneurs des nouvelles filières « vertes";

- Les associations environnementalistes qui jouent le rôle d'un consumérisme vert.

Ces trois types sont ce que Beck appelle les communautés cosmopolites du risque climatique.

\section{Un temps de Grande Politique}

Nietzsche avait pronostiqué l'arrivée d'un temps de Grande Politique. Le changement climatique ou la menace djihadiste font que même les politiques locales sont déterminées par des enjeux globaux, réalisant la prédiction nietzschéenne. Beck était sensible au décalage chez les hommes et les femmes politiques entre la Petite Politique qu'ils pratiquent abondamment et le monde quotidien qui est devenu celui de la Grande Politique.

L'année 2015 multiplie les rendez-vous avec une Grande Politique, mobilisation après les attentats de Paris en janvier, discussions pour préparer la fixation des objectifs du Développement Durable en septembre à l'ONU, et négociations sur le changement climatique en décembre lors de la COP 21. Si les lendemains de l'attentat du 11 septembre 2001 n'avaient pas amené une modernisation réflexive conforme aux prédictions théoriques d'Ulrich Beck, il n'en est plus de même aujourd'hui. La société mondiale du risque génère ces propres "communautés cosmopolites", confirmant les vues prémonitoires d'Ulrich Beck, qui s'est éclipsé discrètement de la vie. 


\section{BIBLIOGRAPHIE}

Beck U. et Boudou B., 2014, « Nationalisme méthodologique-cosmopolitisme méthodologique : un changement de paradigme dans les sciences sociales », Raisons politiques, 54 (2), p. 103-120.

Beck U., Blok A., Tyfield D. et Zhang J.Y., 2013,"Cosmopolitan Communities of Climate Risk : Conceptual and Empirical suggestions for a new research agenda", in Global Networks, 13(1), p. 1-21.

Beck U., Levy D., 2013, "Cosmopolitanized Nations : re-imagining collectivity in World Risk Society", Theory, Culture \& Society, 30(2), p. 3-31.

Beck U. \& Beck-Gernsheim, E., 2013, Distant Love, John Wiley \& Sons.

Beck U., 2013, Non à l'Europe allemande, Paris, Autrement.

Beck U., 2007, "The cosmopolitan condition : Why methodological nationalism fails", in Theory, Culture \& Society, 24 (7-8), p. 286-290.

Beck U., 2007, « La condition cosmopolite et le piège du nationalisme méthodologique », dans Wieviorka M., Les sciences sociales en mutation, Paris, Sciences humaines, p. 223-236.

Beck U., Grande E., 2007, Pour un empire européen. Éditions Flammarion.

Beck U., 2006, Qu'est-ce que le cosmopolitisme?, Paris, Aubier (Première édition en langue allemande, Suhrkamp, 2004).

Beck U., 2003, « La société du risque globalisé revue sous l'angle de la menace terroriste. » in Cahiers internationaux de sociologie, (1), p. 27-33.

Beck U., 2003, Pouvoir et contre-pouvoir à l'ère de la mondialisation, Paris, Aubier (Première édition en langue allemande, Suhrkamp, 2002).

Beck U., 2002, « La dynamique politique de la société mondiale du risque ». Conférence donnée dans le cadre du séminaire Économie de l'environnement et du développement durable, Paris, Institut du développement durable et des relations internationales.

Beck U., 2001, « La politique dans la société du risque » in Revue du MAUSS, (1), p. 376-392.

Beck U., 1998, « Le conflit des deux modernités et la question de la disparition des solidarités : liens personnels, liens collectifs ", in Lien social et politiques, RIAC, (39), p. 15-25.

Beck U., Beck-Gernsheim E., 1995, The normal chaos of love Cambridge, Polity Press.

Beck U., 1994, « D'une théorie critique de la société vers la théorie d'une auto-critique sociale », in Déviance et société, 18 (3), p. 333-344.

Beck U., 1993, « De la société industrielle à la société à risques. Problématique de la survie, structures sociales et éveil d'une conscience écologique », in Revue suisse de sociologie, 19, p. 311-337.

Beck U., 1991, « Die Soziologieund die ökologische Frage » in Berliner Journal fürSoziologie, 1 (3), p. 331-341.

Beck U., 2008, La société du risque : sur la voie d'une autre modernité, Paris, Flammarion (Première édition en langue allemande, 1986). 
AUTEUR

STÉPHANE CALLENS

Stéphane Callens, université d'Artois, LEM UMR 9221 CNRS 